\title{
Late-Onset Encephalopathy Associated With SARS-CoV-2 Infection
}

\author{
Katie Reming ${ }^{\mathrm{a}}$, Keithan Sivakumar ${ }^{\mathrm{b}}$, Negar Moheb ${ }^{\mathrm{b}}$, \\ Ahmad Nizam ${ }^{c}$, Hussam A. Yacoub ${ }^{a}$, b, d
}

\begin{abstract}
Several typical and atypical neurological manifestations of viral pandemics have been reported. Neurological manifestations of the severe acute respiratory syndrome coronavirus 2 (SARS-CoV-2) have recently been reported. In this case report, we present a patient with encephalopathy as a late neurologic manifestation of SARS-CoV-2 infection. The patient initially tested positive for the novel coronavirus after presenting with fever, cough, and altered mental status. The symptoms resolved within 5 - 7 days and the patient was discharged home. He subsequently developed worsening encephalopathy in the absence of respiratory symptoms, required hospitalization, and tested positive for SARS-CoV-2. Complete workup was unrevealing otherwise. We advise clinicians to be aware of late neurological manifestations of coronavirus disease 2019 (COVID-19) including encephalopathy.
\end{abstract}

Keywords: COVID-19; Viral infections; SARS-CoV-2; Encephalopathy

\section{Introduction}

The severe acute respiratory syndrome coronavirus 2 (SARSCoV-2) has rapidly spread, creating a global pandemic. While the most frequently reported symptoms are fever, cough, and dyspnea, it is recognized that the virus can invade the central nervous system causing deleterious effects and a variety of neurological symptoms. A recent retrospective study in Wuhan, China reported that patients with severe infections related to SARS-CoV-2 were more likely to exhibit neurological symptoms than the typical respiratory manifestations that would

Manuscript submitted July 13, 2020, accepted July 20, 2020

Published online December 9, 2020

${ }^{a}$ Morsani College of Medicine, University of South Florida, Tampa, FL, USA bepartment of Neurology, Lehigh Valley Health Network, Allentown, PA, USA

${ }^{\mathrm{c}}$ The Core Institute, Phoenix, AZ, USA

${ }^{\mathrm{d}}$ Corresponding Author: Hussam A. Yacoub, Department of Neurology, Lehigh Valley Health Network, 1250 South Cedar Crest Blvd., Suite 405, Allentown, PA 18103, USA. Email: hussam_a.yacoub@lvhn.org

doi: https://doi.org/10.14740/jnr614 alert health care providers to an underlying SARS-CoV-2 infection [1]. Neurological manifestations include, but are not limited to, headache, disturbed consciousness, encephalopathy, and paresthesias [2, 3]. In April 2020 science brief, the World Health Organization reported that a prior infection with SARS-CoV-2 does not necessarily provide immunity against a second infection [4].

In this report, we present a case of a patient who tested positive for SARS-CoV-2 on two separate occasions, 21 days apart. The patient was initially admitted with respiratory symptoms along with altered mental status, but only with neurological symptoms during the second hospitalization.

\section{Case Report}

A 67-year-old man presented to our facility on April 2, 2020 with fever, cough, lethargy, and worsening hallucinations. Past medical history was significant for hypertension, type 2 diabetes mellitus, and schizoaffective disorder treated with lithium and carbamazepine. Family history was noncontributory. The patient resided in a group home with a known underlying mild intellectual disability. He denied sick contacts or recent travels. Social history was unremarkable for any alcohol or recreational drug use.

On physical examination, the patient was febrile (38.2 ${ }^{\circ} \mathrm{C}$ ) with a blood pressure of $139 / 85 \mathrm{~mm} \mathrm{Hg}$, heart rate of 95 beats $/ \mathrm{min}$, and respiration rate of 16 breaths $/ \mathrm{min}$. Oxygen saturation was $92 \%$ on room air. Pulmonary examination revealed normal effort and breath sounds without wheezes or rales. On neurologic examination, the patient was alert, fully orientated to self, time, and place with no focal neurologic deficits. Laboratory workup included a complete blood count, complete metabolic panel, blood culture and urinalysis, all of which were unremarkable. Nasopharyngeal swab polymerase chain reaction (PCR) for SARS-CoV-2 was positive. A chest X-ray and a computed tomography of the head without contrast were unrevealing. The patient was treated with hydroxychloroquine, $200 \mathrm{mg}$ twice a day for 4 days. Oxygen saturation was monitored throughout the hospitalization with no evidence of hypoxia. He was discharged in a stable condition 4 days after initial presentation. Follow-up telehealth evaluation the following week reported generalized weakness and a low-grade fever, but otherwise no new neurological symptoms.

Three weeks following the initial presentation, the patient 
experienced worsening lethargy in the setting of decreased oral intake, severe encephalopathy, and impaired balance. The rest of the review of systems was unrevealing.

Initial neurological evaluation revealed severe encephalopathy with a Glascow coma score of 11. Patient was awake but inconsistently oriented and unable to follow complex commands. Pupils were equal, round, and reactive to light. Extraocular muscles were intact and no nystagmus was noted. The remaining of the neurological examination was limited. He was found to be hypothermic $\left(33.9{ }^{\circ} \mathrm{C}\right)$. The hypothermia resolved with warmed intravenous fluids and warming blankets, but the patient remained severely encephalopathic. The differential diagnosis for altered mental status included metabolic derangements, toxicity from psychiatric medications in the context of dehydration, and late neurologic manifestation of SARS-CoV-2.

Laboratory workup revealed a white blood cell count (WBC) of $16.21 \times 10^{3} / \mu \mathrm{L}$, hemoglobin of $14.4 \mathrm{~g} / \mathrm{dL}$, and serum glucose of $131 \mathrm{mg} / \mathrm{dL}$. Complete metabolic panel was within normal range. Lithium and carbamazepine levels were mildly elevated to $1.5 \mathrm{mmol} / \mathrm{L}$ and $12.9 \mathrm{mg} / \mathrm{L}$, respectively, but were dose-adjusted, monitored, and subsequently found to be within therapeutic range. No WBCs or nitrates were detected on urinalysis. Cerebrospinal fluid (CSF) analysis revealed a clear and colorless fluid with a mildly elevated protein count of 76 $\mathrm{mg} / \mathrm{dL}$ and a WBC count of $3 / \mu \mathrm{L}$ (all lymphocytes). Complete meningoencephalitis viral PCR panel was negative. SARSCoV-2 PCR testing was not performed on the CSF. An electroencephalogram revealed diffuse slowing consistent with mild encephalopathy. No epileptiform discharges or electrographic seizures were noted. Ten days after admission, patient exhibited decreased inspiratory effort and breath sounds at the lung bases. SARS-CoV-2 was detected in a nasopharyngeal swab by PCR. The patient gradually improved with supportive care, and gradually returned back to his neurologic baseline over the course of several weeks.

\section{Discussion}

There is robust evidence that SARS-CoV-2 can gain access to the central nervous system. PCR confirmed the presence of SARS-CoV-2 in the CSF of infected patients. Preliminary data indicates nervous system involvement in $36 \%$ of known infections [1]. Potential mechanisms of neurologic injury include direct neurotropism, which has been postulated to be the mechanism underlying anosmia/ageusia and encephalitis, systemic inflammatory responses resulting in hypoxic or toxic-metabolic nervous system injury, or a delayed onset autoimmune-mediated mechanism [3]. Symptoms of central nervous system involvement include dizziness and headache and peripheral nervous system involvement causes gustatory and olfactory disturbance [1]. This raises the query of retrograde entry through the olfactory bulb in addition to angiotensin-converting enzyme 2 receptor-mediated access $[3,5]$.

Our case describes the prolonged course of illness in a patient with an active SARS-CoV-2 infection. We propose a cor- relation between the acute viral infection and encephalopathy due to central nervous system involvement. Complete workup for toxic metabolic encephalopathy was unremarkable. While we were unable to confirm the presence of SARS-CoV-2 through CSF PCR testing, this did not rule out encephalopathy due to viral infection. Similar presentations have been documented in cases where SARS-CoV-2 was not detected in the CSF but infection was confirmed with nasopharyngeal swab PCR testing $[6,7]$.

Our case is consistent with previous reports of patients with severe SARS-CoV-2 infections who present with neurologic rather than the typical respiratory symptoms [1]. Further studies are warranted to better understand the pathophysiology and neurological implications of SARS-CoV-2.

\section{Conclusions}

A growing amount of evidence suggests that SARS-CoV-2 has a multitude of neurologic manifestations. To our knowledge, our case is one of the few reported of encephalopathy following a SARS-CoV-2 infection. Patients presenting with symptoms suggestive of acute neurologic manifestations should be evaluated carefully regarding recent symptoms of infection to help identify and prevent late complications of this novel virus.

\section{Acknowledgments}

None to declare.

\section{Financial Disclosure}

No funding was received. None of the authors have disclosures relevant to this manuscript.

\section{Conflict of Interest}

None to declare.

\section{Informed Consent}

Not applicable.

\section{Author Contributions}

All authors participated in data collection, data interpretation and editing the manuscript.

\section{Data Availability}

The authors declare that data supporting the findings of this 
study are available within the article.

\section{References}

1. Mao L, Jin H, Wang M, Hu Y, Chen S, He Q, Chang J, et al. Neurologic manifestations of hospitalized patients with coronavirus disease 2019 in Wuhan, China. JAMA Neurol. 2020;77(6):683-690.

2. Asadi-Pooya AA, Simani L. Central nervous system manifestations of COVID-19: A systematic review. J Neurol Sci. 2020;413:116832.

3. Wu Y, Xu X, Chen Z, Duan J, Hashimoto K, Yang L, Liu $\mathrm{C}$, et al. Nervous system involvement after infection with COVID-19 and other coronaviruses. Brain Behav Im- mun. 2020;87:18-22.

4. https://www.who.int/news-room/commentaries/detail/ immunity-passports-in-the-context-of-covid-19.

5. Li YC, Bai WZ, Hashikawa T. The neuroinvasive potential of SARS-CoV2 may play a role in the respiratory failure of COVID-19 patients. J Med Virol. 2020;92(6):552555.

6. Yin R, Feng W, Wang T, Chen G, Wu T, Chen D, Lv T, et al. Concomitant neurological symptoms observed in a patient diagnosed with coronavirus disease 2019. J Med Virol. 2020;92(10):1782-1784.

7. Moriguchi T, Harii N, Goto J, Harada D, Sugawara H, Takamino J, Ueno M, et al. A first case of meningitis/encephalitis associated with SARS-Coronavirus-2. Int J Infect Dis. 2020;94:55-58. 